\title{
Observer Design for Motorcycle's Lean and Steering Dynamics Estimation: a Takagi-Sugeno Approach
}

\author{
Dalil Ichalal, Hichem Arioui and Saïd Mammar
}

\begin{abstract}
In this paper, a nonlinear motorcycle model is considered in order to estimate both the lean and steering dynamics. The model is transformed into a Takagi-Sugeno (T-S) form using the well-known sector nonlinearity approach. The first contribution of this work is the exactness of the obtained T-S model compared to the considered nonlinear model, where the weighting functions of the T-S model depend on unmeasured state variables. A novel approach to construct a nonlinear unknown input fast observer is proposed. The objective is the simultaneous reconstruction of the state variables and the rider's torque. The observer's convergence is studied using Lyapunov theory guaranteeing boundedness of the state and unknown input estimation errors which is expressed by the Input to State Practical Stability (ISpS). Stability conditions are then expressed in terms of Linear Matrix Inequalities (LMI). Finally, simulation results are provided to confirm the suitability of the proposed nonlinear observer.
\end{abstract}

\section{INTRODUCTION}

Currently, powered two-wheeled (PTW) vehicles is a mean of transport increasingly sought after, especially for the opportunities it offers to sidestep traffic congestion. This increase in vehicle fleet to PTW is accompanied by the blast of the number of accidents. For a long time, industrial societies record on their roadways thousands of deaths and other fatalities per year. Road safety institutions have launched several preventive actions (radar, tickets, etc.) and research programs headed for safety systems, mainly for cars. Some of these steps have brought their results, since the number of fatalities recorded a significant decrease over the past few years. However, if the number of fatalities experiences an overall decrease, the PTW remains a particularly dangerous transportation, [13], [2].

Development of safety systems for cars has reached a certain maturity. Unfortunately, for motorcycles it is not the case. The most prominent example is that of the ABS, that exists for 20 years, but is not still standard equipment for PTW and its use remains marginal, [7]. The direct transposition of security systems, from cars to motorcycles, is not obvious because of the complex motorcycle dynamics (highly nonlinear, [5], [8]), for example. In addition, ahead of the design of a safety system, we must ensure the availability of the relevant dynamic states in order to quantify the risk (loss of control, skidding, etc.). PTW dynamics can be estimated through suitable sensors and/or observers. The first way is generally avoided for several reasons: price, noise measurement, feasibility, etc. On the other hand, there are

All the authors are with the IBISC Laboratory, EvryVal-d'Essonne University, 40, rue de Pelvoux, 91020 Evry Courcouronne Cedex \{dalil.ichalal, hichem.arioui, said.mammar\}@ibisc.univ-evry.fr only few works dealing with the problem of motorcycle state observation.

In the literature, most studies have mainly concerned the estimation of the lean dynamics unlike the steering one. Different techniques have been proposed to estimate the roll angle: for example, frequency separation filtering [4] or extended Kalman filters, [18]. These techniques, performed under restrictive assumptions (dynamic steering is neglected, tire-road forces are linear, etc.), are not robust against the variations of the forward velocity.

The topic of estimation of the steering angle (not the rider's torque reconstruction) is not well covered in literature as the lean angle estimation problem. However, a few results have been obtained in [6], where an LPV observer has been used to design single-sensor control strategies for a semi-active steering damper. The approach is a simple gain scheduling for an LTI motorcycle model under three constant forward velocities $(50,100$ and $140 \mathrm{~m} / \mathrm{s}$ ). Unfortunately, no guarantees for stability or convergence of the LPV observer are given outwards these constant velocities.

To the best knowledge of the authors, the simultaneous estimation of the lean and the steering dynamics (rider's torque) have never been addressed.

\section{Problem Statement}

Our long term objective points the identification of all pertinent inputs and dynamic states improving the risk quantification of the loss-of-control during cornering. Indeed, inadequate cornering is responsible for most motorcycle fatalities, especially for single motorcycle crashes. For safe cornering, riders should respect: 1) a suitable speed before starting the corner, 2) the road conditions (under weak friction) and 3) weather conditions do not allow optimal visibility when riding. Early warning systems are based generally on related work carried out for standard cars [15]. The goal is the synthesis of a function estimating the maximum safe speed at which a vehicle can be kept stable on the road while moving at a constant longitudinal velocity on a circular section. This velocity depends, among other factors, on the lateral friction $\mu_{\text {lat }}$ whose computation involves all the dynamic states of the PTW and a good modeling of the tireroad contact [15]. This makes the success of such warning systems strongly dependent on the availability of dynamic states of the motorcycle. Correlating this fact with the highly nonlinear dynamics, standard observation approaches are questionable and less efficient. To answer this real challenge, we address in this paper a nonlinear observer synthesis based on Takagi-Sugeno approach for PTW vehicles. 
Takagi-Sugeno fuzzy structure is one of the most interesting approach to model nonlinear behaviors as proven in recent years [16], [17]. Indeed, it offers a way to represent nonlinear complex behaviors by a more tractable mathematical formulation inspired by linear models. It consists on decomposing the operating state space on several regions and each region is modeled by a linear model. Thanks to nonlinear weighting functions which satisfy the convex sum property, the overall nonlinear behavior of a system is characterized in a compact set of the state space. In recent years, some works are dedicated to the study of nonlinear systems via T-S models, especially in observers design [9], [3], [1]. The proposed observer in this paper is inspired from the linear adaptive one in [19] and adapted to T-S models with unmeasurable premise variables which constitutes an open and interesting field of research. Indeed, the most developed work in the literature are limited only to the case of T-S models with measurable premise variables. T-S models with unmeasurable premise variables have an interesting properties, namely, the ability to transform a general nonlinear model into a T-S model by the use of nonlinear sector nonlinearity transformation with no loss of information (see [10] and references therein), and it is pointed out in [20] that T-S models with unmeasurable premise variables can describe a wider class of nonlinear systems.

The paper is organized as follows: Section III presents the lateral dynamics model of a motorcycle and a TakagiSugeno formulation of the considered model. In Section IV, a unknown input and state observer for estimating the motorcycle lateral dynamics and the steering torque is synthesized. The convergence of the observer is studied with Lyapunov theory and an optimization problem under LMI constraints is provided to design the observer in such a way to guarantee the ISpS Property which illustrates the boundedness of the state and unknown input errors. Finally, Section V provides some simulations results and discussions on the proposed observer.

\section{MOTORCYCLE MODEL DESCRIPTION}

\section{A. Nonlinear model of the motorcycle}

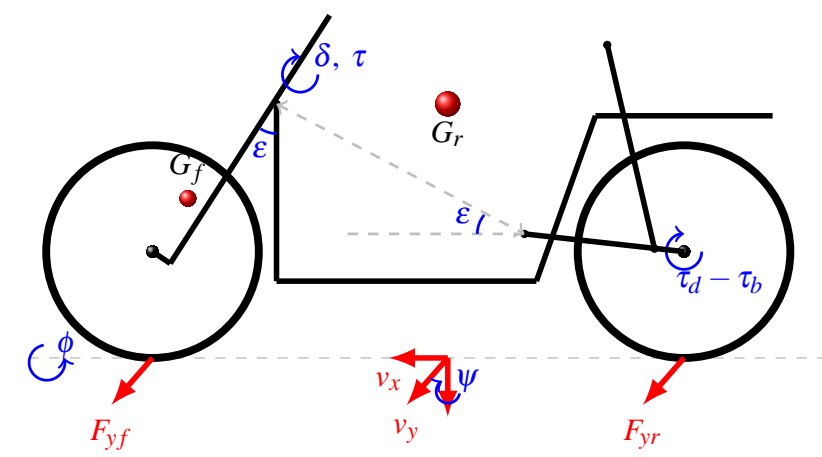

Fig. 1. Geometrical representation of the Sharp's motorcycle model

The lateral dynamics of a motorcycle are represented by a model with four equations [14], [15] describing the lateral motion, due essentially to the effect of lateral forces from the front and rear wheels $\left(F_{y f}\right.$ et $\left.F_{y r}\right)$ and the yaw and roll motions under rider's steering actions. The study of such a model aims to reconstruct the dynamic states of a motorcycle in cornering situation.

These movements expressed by the following equations (all the parameters are defined in Annex 2):

- Lateral motion

$$
M \dot{v}_{y}+M_{f} k \ddot{\psi}+\left(M_{f} j+M_{r} h\right) \ddot{\phi}+M_{f} e \ddot{\delta}=F_{y f}+F_{y r}
$$

- Roll motion

$$
\left(M_{f} j+M_{r} h\right) \dot{v}_{y}+a_{1} \ddot{\phi}+a_{2} \ddot{\psi}+a_{3} \ddot{\delta}+a_{4} v_{x} \dot{\psi}+a_{4} v_{x} \dot{\delta}=\sum \begin{gathered}
M_{x} \\
(2)
\end{gathered}
$$

- Yaw motion

$$
M_{f} k\left(\dot{v}_{y}+v_{x} \dot{\psi}\right)+a_{2} \ddot{\phi}+b_{1} \ddot{\psi}+b_{2} \ddot{\delta}-b_{3} v_{x} \dot{\phi}-a_{5} v_{x} \dot{\delta}=\sum M_{z}
$$

- Steering motion

$$
M_{f} e \dot{v}_{y}+a_{3} \ddot{\phi}+c_{1} \ddot{\psi}+c_{2} \ddot{\delta}-a_{5} v_{x} \dot{\phi}+c_{3} v_{x} \dot{\psi}=\sum M_{S}
$$

where:

$$
\begin{aligned}
\sum M_{x} & =\left(M_{f} j+M_{r} h\right) g \sin (\phi)+\left(M_{f} e g-\eta F_{z f}\right) \sin (\delta) \\
\sum M_{z} & =l_{f} F_{y f}-l_{r} F_{y r}-\tau \cos (\varepsilon) \\
\sum M_{s} & =\left(M_{f} e g-\eta F_{z f}\right) \sin (\phi) \\
& +\left(M_{f} e g-\eta F_{z f}\right) \sin (\varepsilon) \sin (\delta)+\tau
\end{aligned}
$$

The lateral forces $F_{y f}$ and $F_{y r}$ acting, respectively, on the front and rear wheels depend on the sideslip angles $\alpha_{f}$ and $\alpha_{r}$ and camber angles $\gamma_{f}$ and $\gamma_{r}$, are expressed by:

$$
\left\{\begin{array}{l}
F_{y f}=-C_{f 1} \alpha_{f}+C_{f 2} \gamma_{f} \\
F_{y r}=-C_{r 1} \alpha_{r}+C_{r 2} \gamma_{r}
\end{array}\right.
$$

where

$$
\left\{\begin{aligned}
\alpha_{f} & =\left(\frac{v_{y}+l_{f} \dot{\psi}-\eta \dot{\delta}}{v_{x}}\right)-\delta \cos (\varepsilon) \\
\alpha_{r} & =\left(\frac{v_{y}-l_{r} \dot{\psi}}{v_{x}}\right)
\end{aligned}\right.
$$

and

$$
\left\{\begin{array}{l}
\gamma_{f}=\phi+\delta \sin (\varepsilon) \\
\gamma_{r}=\phi
\end{array}\right.
$$

In this work, a normal riding is considered (without taking into account the limit situations) which justifies the linear form of the lateral forces $F_{y f}$ and $F_{y r}$ with respect to both sideslip and camber angles.

By replacing the mathematic expressions of the forces in the dynamics model and by choosing the state vector as $x(t)=\left[\begin{array}{llllll}v_{y} & \dot{\psi} & \dot{\phi} & \phi & \dot{\delta} & \delta\end{array}\right]^{T}$, the system is rewritten as follows:

$$
E \dot{x}(t)=A\left(x(t), v_{x}\right) x(t)+B \tau(t)
$$

where $E$ is a constant nonsingular matrix, $B$ is a constant matrix and $A(x)$ is a nonlinear matrix given by:

$$
A(x)=\left(\begin{array}{cccc}
a_{11} & a_{12}\left(v_{x}\right) & 0 & a_{14} \\
a_{21} & a_{22}\left(v_{x}\right) & a_{23}\left(v_{x}\right) & a_{24} \\
0 & a_{32}\left(v_{x}, \dot{\phi}\right) & 0 & a_{34}\left(v_{x}, \phi\right) \\
0 & 0 & 1 & 0 \\
a_{51}\left(v_{x}\right) & a_{52}\left(v_{x}\right) & a_{53}\left(v_{x}\right) & a_{54}\left(v_{x}, \phi\right) \\
0 & 0 & 0 & 0
\end{array}\right.
$$




$$
\begin{aligned}
& \left.\begin{array}{cc}
a_{15}\left(v_{x}\right) & a_{16} \\
a_{25}\left(v_{x}\right) & a_{26}\left(v_{x}\right) \\
a_{35}\left(v_{x}\right) & a_{36}\left(v_{x}\right) \\
0 & 0 \\
a_{54}\left(v_{x}\right) & a_{56}\left(v_{x}\right) \\
1 & 0
\end{array}\right) \\
& E=\left(\begin{array}{cccccc}
M & e_{12} & e_{13} & 0 & e_{15} & 0 \\
e_{12} & e_{22} & e_{23} & 0 & e_{25} & 0 \\
e_{13} & e_{23} & e_{33} & 0 & e_{35} & 0 \\
0 & 0 & 0 & 1 & 0 & 0 \\
e_{15} & e_{25} & e_{35} & 0 & e_{55} & 0 \\
0 & 0 & 0 & 0 & 0 & 1
\end{array}\right) \\
& B=\left(\begin{array}{cccccc}
0 & -\cos (\varepsilon) & 0 & 0 & 1 & 0
\end{array}\right)^{T}
\end{aligned}
$$

The components $a_{i j}$ and $e_{i j}$ of, respectively, the matrix $A(x)$ and $E$ are given in Annex 1.

\section{B. Exact T-S model of a motorcycle nonlinear model}

In order to express the model in T-S fuzzy structure, let us consider the following nonlinearities:

$$
z_{1}=v_{x}, \quad z_{2}=\frac{1}{v_{x}}, \quad z_{3}=\frac{\sin (\phi)}{\phi}, \quad z_{4}=\dot{\phi}
$$

It is important to notice that the motorcycle, contrarily to a standard vehicle with four wheels, is stable only for a range longitudinal velocities $v_{x}$. Consequently, taking $v_{x}$ in the interval where the motorcycle is stable, the bounds of the premise variables are given by:

$$
\begin{aligned}
& z_{1}^{\min } \leq z_{1} \leq z_{1}^{\max } \\
& z_{2}^{\min } \leq z_{2} \leq z_{2}^{\max } \\
& z_{3}^{\min } \leq z_{3} \leq z_{3}^{\max } \\
& z_{4}^{\min } \leq z_{4} \leq z_{4}^{\max }
\end{aligned}
$$

By following the well-known sector nonlinearity approach (see [17] for more details), the obtained model is:

$$
E \dot{x}(t)=\sum_{i=1}^{16} \mu_{i}\left(v_{x}, x\right) \mathscr{A}_{i} x(t)+\mathscr{B} \tau(t)
$$

The number 16 of sub-models comes from the fact that 4 nonlinearities are chosen (see [17] for more details). Since the matrix $E$ is nonsingular, its inverse $E^{-1}$ exists and allows to write the model (13) in the form:

$$
\dot{x}(t)=\sum_{i=1}^{16} \mu_{i}\left(v_{x}, x\right) A_{i} x(t)+B \tau(t)
$$

where $A_{i}=E^{-1} \mathscr{A}_{i}$ and $B=E^{-1} \mathscr{B}$.

\section{Measurement equation}

Sensors generally available allow to measure different state variables, as the roll and yaw rates $\dot{\phi}, \dot{\psi}$ and both the steering angle and the steering angle rate $\delta$ and $\dot{\delta}$, which leads to the output equation:

$$
y(t)=C x(t)
$$

where:

$$
C=\left(\begin{array}{llllll}
0 & 1 & 0 & 0 & 0 & 0 \\
0 & 0 & 1 & 0 & 0 & 0 \\
0 & 0 & 0 & 0 & 1 & 0 \\
0 & 0 & 0 & 0 & 0 & 1
\end{array}\right)
$$

\section{OBSERVER DESIGN}

The following lemma is used in the proof of the observer's convergence study.

Lemma 1: Consider two matrices $X$ and $Y$ with appropriate dimensions, $G$ a positive definite matrix. The following property holds

$$
X^{T} Y+Y^{T} X \leq X^{T} G X+Y^{T} G^{-1} Y \quad G>0
$$

The objective of this section is to provide a new approach in order to design observers for T-S fuzzy systems with unmeasurable premise variables which is the case for the considered motorcycle model. The observer aims to estimate, simultaneously, the state variables and the rider's torque, especially, the lateral velocity $v_{y}$, the roll angle $\phi$ and the steering torque $\tau$ which constitute the most important variables in synthesizing risk function for riding assistance.

The following nonrestrictive assumptions can be made

Assumption 1: In the remaining it is supposed that

- the state $x(t)$ is bounded (stable or stabilized motorcycle)

- $\operatorname{rank}(C B)=n_{u}$ ( $n_{u}$ is the dimension of the unknown input vector)

- the torque derivative $\dot{\tau}(t)$ is bounded by $\tau_{1 \max }$ In the case of the considered motorcycle model, $\operatorname{dim}(\tau)=$ $n_{u}=1$. The third assumption is not restrictive since the variation of the rider's steering torque is bounded.

\section{A. State estimation}

Let us consider the T-S model given by the equations (14) and (15). The state observer is then given by the following equations:

$$
\left\{\begin{array}{l}
\dot{\hat{x}}(t)=\sum_{i=1}^{16} \mu_{i}\left(v_{x}, \hat{x}\right)\left(A_{i} \hat{x}(t)+L_{i}(y(t)-\hat{y}(t))+B \hat{\tau}(t)\right. \\
\hat{y}(t)=C \hat{x}(t) \\
\dot{\hat{\tau}}(t)=\Gamma F\left(\dot{e}_{y}(t)+\sigma e_{y}(t)\right) \\
e_{y}(t)=y(t)-\hat{y}(t)
\end{array}\right.
$$

Let us consider the state estimation error $e(t)=x(t)-\hat{x}(t)$ and torque estimation errors $e_{\tau}(t)=\tau(t)-\hat{\tau}(t)$. The matrices $L_{i}, F$ and the scalars $\Gamma$ and $\sigma$, of the observer, are to be determined in such a way to ensure state and unknown input estimation errors with a minimal bound to have an accurate estimations.

Using equations (14), (15) and (17), the state estimation error obeys the following differential equation:

$$
\dot{e}(t)=\sum_{i=1}^{16} \mu_{i}\left(v_{x}, \hat{x}\right) \Phi_{i} e(t)+B e_{\tau}(t)+\Delta(t)
$$

where:

$$
\Phi_{i}=A_{i}-L_{i} C
$$

and:

$$
\Delta(t)=\sum_{i=1}^{16}\left(\mu_{i}\left(v_{x}, x\right)-\mu_{i}\left(v_{x}, \hat{x}\right)\right) A_{i} x(t)
$$

Notice that if the state estimation errors converge to zero, the term $\Delta(t)$ converges also towards zero. In addition, since the 
weighting functions are bounded and the state vector $x(t)$ is also bounded (see assumption 1), the term $\Delta(t)$ is then bounded. The objective is to design the matrices $L_{i}, F$ and the scalars $\Gamma$ and $\sigma$ guaranteeing an accurate estimation of the state and the unknown input by minimizing the bound of the state and unknown estimation errors.

\section{B. Observer's convergence study}

From Assumption 1 and the fact that the functions $\mu_{i}$ are bounded, the term $\Delta(t)$ is bounded. Indeed, the system is stable which provides bounded states for bounded input $\tau(t)$. Under bounded perturbation term $\Delta(t)$, the observer (17) is synthesized by solving the optimization problem under LMI constraints given in the Theorem 1.

Definition 1: ([12], [11]) The system (18) verify the Input To State Practical Stability (ISpS) if there exists a $\mathscr{K} \mathscr{L}$ function $\beta: \mathbb{R}^{6+1} \times \mathbb{R} \longrightarrow \mathbb{R}$, a $\mathscr{K}$ function $\alpha: \mathbb{R} \longrightarrow \mathbb{R}$ and a constant $d$ such that for each input $\Delta(t)$ satisfying $\|\Delta(t)\|_{\infty}<\infty$ and each initial conditions $e(0)$, the trajectory of (18) associated to $e(0)$ and $\Delta(t)$ satisfies

$$
\|e(t)\|_{2} \leq \beta(\|e(0)\|, t)+\alpha\left(\|\Delta(t)\|_{\infty}\right)+d
$$

Theorem 1: Under the Assumption 1, given a positive scalars $\sigma$ and $\alpha$ and $a \in[0,1]$, if there exists a symmetric and positive definite matrix $P$, gain matrices $M_{i}$ and positive scalars $G, \gamma, \eta$ and $S$ solution to the following optimization problem, $i=1, \ldots, 16$

$$
\min _{P, M_{i}, \eta, \gamma} a \eta+(1-a) \gamma
$$

s.t.

$$
\begin{gathered}
B^{T} P=F C \\
\left(\begin{array}{ccc}
\Omega_{i}+\alpha P & -\frac{1}{\sigma} \Phi_{i}^{T} P B-P B & P \\
-\frac{1}{\sigma} B^{T} P \Phi_{i}-B^{T} P & \Psi+\alpha \frac{S}{\sigma} & -\frac{1}{\sigma} B^{T} P \\
P & -\frac{1}{\sigma} P B & -\gamma
\end{array}\right)<0 \\
\left(\begin{array}{cc}
\alpha \eta I & Q \\
Q & \alpha \eta I
\end{array}\right)>0 \\
Q \geq I
\end{gathered}
$$

where:

$$
\begin{aligned}
\Omega_{i} & =A_{i}^{T} P+P A_{i}-M_{i} C-C^{T} M_{i}^{T} \\
\Psi & =-\frac{2}{\sigma} B^{T} P B+\frac{1}{\sigma} G \\
Q & =\operatorname{diag}\left(P, \frac{S}{\sigma}\right)
\end{aligned}
$$

and $\Gamma=S^{-1}$, then the state and torque estimation errors are bounded. The gains of the observer are computed from $L_{i}=P^{-1} M_{i}$ and $F$ is obtained directly by solving the above optimization problem. The attenuation level of the transfer from $\Delta(t)$ to state and unknown input estimation errors is bounded and given by the quantity $\sqrt{\frac{\lambda_{\max }(Q) \gamma}{\lambda_{\min }(Q) \alpha}}$. In addition, If $\Delta(t)=0$ the state and unknown input estimation errors (norms of errors) converge to a set around the origin with a size defined by the quantity $\sqrt{\frac{\lambda_{\max }(Q) \delta}{\lambda_{\min }(Q) \alpha}}$ where:

$$
\delta=\frac{1}{\sigma} \tau_{1 \max }^{2} \lambda_{\max }\left(\Gamma^{-1} G^{-1} \Gamma^{-1}\right)
$$

The parameter $\Gamma$ is then chosen sufficiently large in order to have a minimal value of $\delta$ which guaranteeing a more accurate estimations. Otherwise, if $\Delta(t) \neq 0$, the state estimate errors guaranty the Input To State Practical Stability (ISpS) given by the following property

$$
\|e(t)\|_{2} \leq \sqrt{\frac{\lambda_{\max }(Q)}{\lambda_{\min }(Q)}}\left(e^{-\frac{\alpha}{2} t}+\sqrt{\frac{\gamma}{\alpha}}\|\Delta(t)\|_{\infty}+\sqrt{\frac{\delta}{\alpha}}\right)
$$

Proof: In order to prove the convergence of the state and torque estimation errors, let us consider the following Lyapunov function:

$$
V(t)=e^{T}(t) P e(t)+\frac{1}{\sigma} e_{\tau}^{T}(t) \Gamma^{-1} e_{\tau}(t)
$$

where $P=P^{T}>0$ and $\Gamma>0$. In the framework of the motorcycle model, the dimension of $\tau$ is 1 then the parameter $\Gamma$ is a scalar. (For more general case, we have $\Gamma \in \mathbb{R}^{n_{u}}$ where $n_{u}$ is the dimension of the unknown input vector).

According to the equation (18), the time derivative of $V(t)$ is given by:

$$
\begin{aligned}
\dot{V}(t) & =\sum_{i=1}^{16} \mu_{i}\left(v_{x}, \hat{x}(t)\right) e^{T}(t)\left(\Phi_{i}^{T} P+P \Phi_{i}\right) e(t) \\
& +\frac{2}{\sigma} e_{\tau}^{T}(t) \Gamma^{-1} \dot{e}_{\tau}(t)+2 e^{T}(t) P \Delta(t)+2 e_{\tau}^{T}(t) B^{T} P e(t)
\end{aligned}
$$

Knowing that $\dot{e}_{\tau}(t)=\dot{\tau}-\dot{\hat{\tau}}(t)$ and given the expression of $\dot{\hat{\tau}}(t)$ (17), the time derivative of the Lyapunov function (32) becomes:

$$
\begin{aligned}
\dot{V}(t) & =\sum_{i=1}^{16} \mu_{i}\left(v_{x}, \hat{x}(t)\right) e^{T}(t) \Omega_{i} e(t) \\
& -\frac{2}{\sigma} e_{\tau}^{T}(t) F\left(\dot{e}_{y}(t)+\sigma e_{y}(t)\right) \\
& +\frac{2}{\sigma} e_{\tau}^{T}(t) \Gamma^{-1} \dot{\tau}(t)+2 e^{T}(t) P \Delta(t) \\
& +2 e_{\tau}^{T}(t) B^{T} P e(t)
\end{aligned}
$$

where $\Omega_{i}=\Phi_{i}^{T} P+P \Phi_{i}$. Using the output equations, we have $e_{y}(t)=C e(t)$ and $\dot{e}_{y}(t)=C \dot{e}$, then, equation (33) can be rewritten under the following form:

$$
\begin{aligned}
\dot{V}(t) & =\sum_{i=1}^{16} \mu_{i}\left(v_{x}, \hat{x}(t)\right)\left(e^{T}(t) \Omega_{i} e(t)-\frac{2}{\sigma} e_{\tau}^{T}(t) F C \Phi_{i} e(t)\right) \\
& -\frac{2}{\sigma} e_{\tau}^{T}(t) F C \Delta(t)-2 e_{\tau}^{T}(t) F C e(t)+\frac{2}{\sigma} e_{\tau}^{T}(t) \Gamma^{-1} \dot{\tau}(t) \\
& -\frac{2}{\sigma} e_{\tau}^{T}(t) F C B e_{\tau}(t)+2 e^{T}(t) P \Delta(t)
\end{aligned}
$$

Using lemma 1 and the fact that the first derivative of $\tau$ is bounded by $\tau_{1 \max }$ (assumption 1 ), we obtain

$$
\begin{aligned}
& \frac{2}{\sigma} e_{\tau}^{T}(t) \Gamma^{-1} \dot{\tau}(t) \\
\leq & \frac{1}{\sigma} e_{\tau}^{T}(t) G e_{\tau}(t)+\frac{1}{\sigma} \dot{\tau}^{T}(t) \Gamma^{-1} G^{-1} \Gamma^{-1} \dot{\tau}(t) \\
\leq & \frac{1}{\sigma} e_{\tau}^{T}(t) G e_{\tau}(t)+\frac{1}{\sigma} \tau_{1 \max }^{2} \lambda_{\max }\left(\Gamma^{-1} G^{-1} \Gamma^{-1}\right)
\end{aligned}
$$


and using assumption 1 . It is possible to obtain matrices $F$ and $P$ such that $B^{T} P=F C$ holds. The time derivative of the Lyapunov function (34) is then bounded as follows

$$
\begin{aligned}
\dot{V}(t) & \leq \sum_{i=1}^{16} \mu_{i}\left(v_{x}, \hat{x}(t)\right) e^{T}(t) \Omega_{i} e(t)-\frac{2}{\sigma} e_{\tau}^{T}(t) B^{T} P \Phi_{i} e(t) \\
& -\frac{2}{\sigma} e_{\tau}^{T}(t) B^{T} P \Delta(t)-2 e_{\tau}^{T}(t) B^{T} P e(t) \\
& -\frac{2}{\sigma} e_{\tau}^{T}(t) B^{T} P B e_{\tau}(t)+\frac{1}{\sigma} e_{\tau}^{T}(t) G e_{\tau}(t) \\
& +\frac{1}{\sigma} \tau_{1 \max }^{2} \lambda_{\max }\left(\Gamma^{-1} G^{-1} \Gamma^{-1}\right)+2 e^{T}(t) P \Delta(t)
\end{aligned}
$$

Let us define $\tilde{x}(t)=\left[\begin{array}{lll}e^{T}(t) & e_{\tau}(t) & \Delta(t)\end{array}\right]^{T}$. The inequality (36) is equivalent to:

$$
\begin{aligned}
\dot{V}(t) & \leq \tilde{x}^{T}(t) \sum_{i=1}^{16} \mu_{i}\left(v_{x}, \hat{x}(t)\right) \Xi_{i} \tilde{x}(t) \\
& -\alpha e_{a}^{T}(t) Q e_{a}(t)+\gamma \Delta^{T}(t) \Delta(t)+\delta
\end{aligned}
$$

where $\alpha$ and $\gamma$ are positive scalars, $Q=\operatorname{diag}\left(P, \frac{\Gamma^{-1}}{\sigma}\right), e_{a}(t)=$ $\left[\begin{array}{ll}e^{T}(t) & e_{\tau}(t)\end{array}\right]^{T}$ and

$$
\Xi_{i}=\left(\begin{array}{ccc}
\Omega_{i}+\alpha P & -\frac{1}{\sigma} \Phi_{i}^{T} P B-P B & P \\
-\frac{1}{\sigma} B^{T} P \Phi_{i}-B^{T} P & \Psi+\alpha \frac{\Gamma^{-1}}{\sigma} & -\frac{1}{\sigma} B^{T} P \\
P & -\frac{1}{\sigma} P B & -\gamma^{2}
\end{array}\right)
$$

and

$$
\begin{gathered}
\delta=\frac{1}{\sigma} \tau_{1 \max }^{2} \lambda_{\max }\left(\Gamma^{-1} G^{-1} \Gamma^{-1}\right) \\
\Psi=-\frac{2}{\sigma} B^{T} P B+\frac{1}{\sigma} G \\
\Omega_{i}=\Phi_{i}^{T} P+P \Phi_{i}
\end{gathered}
$$

Now, if $\tilde{x}^{T}(t) \sum_{i=1}^{16} \mu_{i}\left(v_{x}, \hat{x}(t)\right) \Xi_{i} \tilde{x}(t)<0$ then the inequality (37) can be bounded as follows

$$
\dot{V}(t) \leq-\alpha e_{a}^{T}(t) Q e_{a}(t)+\gamma \Delta^{T}(t) \Delta(t)+\delta
$$

which is equivalent to

$$
\dot{V}(t) \leq-\alpha V(t)+\gamma \Delta^{T}(t) \Delta(t)+\delta
$$

It follows

$$
\begin{aligned}
V(t) & \leq V(0) e^{-\alpha t}+\gamma \int_{0}^{t} e^{-\alpha(t-s)}\|\Delta(s)\|_{2}^{2} d s \\
& +\delta \int_{0}^{t} e^{-\alpha(t-s)} d s \\
& \leq V(0) e^{-\alpha t}+\frac{\gamma}{\alpha}\|\Delta(t)\|_{\infty}^{2}+\frac{\delta}{\alpha}
\end{aligned}
$$

Knowing that $\lambda_{\min }(Q)\left\|e_{a}(t)\right\|_{2}^{2} \leq V(t) \leq \lambda_{\max }(Q)\left\|e_{a}(t)\right\|_{2}^{2}$, where:

$$
Q=\left(\begin{array}{cc}
P & 0 \\
0 & \frac{\Gamma^{-1}}{\sigma}
\end{array}\right)
$$

one obtains the inequality

$$
\left\|e_{a}(t)\right\|_{2}^{2} \leq \frac{\lambda_{\max }(Q)}{\lambda_{\min }(Q)}\left(e^{-\alpha t}+\frac{\gamma}{\alpha}\|\Delta(t)\|_{\infty}^{2}+\frac{\delta}{\alpha}\right)
$$

By using the square root on (44), on obtains

$$
\left\|e_{a}(t)\right\|_{2} \leq \sqrt{\frac{\lambda_{\max }(Q)}{\lambda_{\min }(Q)}}\left(e^{-\frac{\alpha}{2} t}+\sqrt{\frac{\gamma}{\alpha}}\|\Delta(t)\|_{\infty}+\sqrt{\frac{\delta}{\alpha}}\right)
$$

According to Lyapunov formulation of Input To State Practical Stability (ISpS), the state and unknown input errors converge to a region which will be minimized in order to achieve a more accurate estimation of the states of the vehicle and the torque applied on the handlebar. This ball is smaller as the constant $\delta$ and the attenuation level of the transfer from $\Delta(t)$ to the state estimation errors are smaller. To enhance the performances of the observer, a minimal values of these quantities are obtained by the following reasoning: Let us consider the quantity $\sqrt{\frac{\lambda_{\max }(Q)}{\lambda_{\min }(Q) \alpha}} \leq \sqrt{\eta}$ where $\eta$ is a positive scalar. It is then sufficient to minimize the term $\eta$ and assuming $\lambda_{\min }(Q) \geq 1(Q>I)$, on obtains:

$$
\sqrt{\frac{\lambda_{\max }(Q)}{\alpha}} \leq \sqrt{\eta}
$$

which is transformed easily into:

$$
(\alpha \eta)^{2} I-Q^{T} Q>0
$$

Using Shur's complement lemma:

$$
\left(\begin{array}{cc}
\alpha \eta I & Q \\
Q & \alpha \eta I
\end{array}\right)>0
$$

The second quantity $\sqrt{\frac{\lambda_{\max }(Q) \delta}{\lambda_{\min }(Q) \alpha}}$ is minimized by choosing the parameters $\alpha$ and $G$ sufficiently large for minimizing the term $\delta$ and the the term $\sqrt{\frac{\lambda_{\max }(Q)}{\lambda_{\min }(Q)}}$ is treated above. Finally, the bound of this term will be $\sqrt{\delta / \alpha}$ multiplied by $\eta$. Always in the purpose of minimizing the two quantities, in theorem 1, the chosen objective function is a linear combination between $\eta$ and $\gamma$. By choosing the change of variables $S=\Gamma^{-1}$ and $M_{i}=P L_{i}$, the linear matrix inequalities in theorem 1 are obtained.

\section{SIMULATION RESULTS}

The nonlinear system, including longitudinal and lateral dynamics of two-wheeled vehicle is used. It requires three inputs: the rider's steering torque applied on the handlebars $\tau$ (see Figure 2) and the angular velocities of both front and rear wheels $\omega_{f}$ and $\omega_{r}$. The observer estimating the lateral dynamics and steering torque using only the measured states $\dot{\psi}, \dot{\phi}$ given by the inertial unit and $\dot{\delta}$ and $\delta$ obtained from a suitable encoder. The gains of the observer were calculated by solving the optimization problem under LMI constraints proposed in theorem 1 . The obtained attenuation level, from the term $\Delta(t)$ to the state and unknown input estimation errors is $\gamma=2.6119$. The initial conditions of the system are $x(0)=\left[\begin{array}{llllll}0 & 0 & 0 & 0 & 0 & 0\end{array}\right]^{T}$ and those of the observer are $\hat{x}(0)=\left[\begin{array}{llllll}1 & 1 & 0.1 & 1 & 0 & 0.1\end{array}\right]^{T}$. The adaptation law 
providing an estimation of the steering torque is designed in such a way to have a fast convergence of $\hat{\tau}(t)$ to $\tau(t)$. The chosen parameters are $\alpha=1, \sigma=5$ and $a=0.99$. If $\Delta(t)=0$, the state and unknown input estimation errors $e(t)$ and $e_{a}(t)$ converge to a ball around the origin having the size $\sqrt{\frac{\lambda_{\max }(Q) \delta}{\lambda_{\min }(Q) \alpha}} \approx 0.1$, for $\tau_{1 \text { max }}=40$. In the steady state $\|\Delta(t)\|_{\infty}$ is less than 0.07 , the attenuation of $\Delta(t)$ is then given by $\sqrt{\frac{\lambda_{\max }(Q)}{\lambda_{\min }(Q) \alpha}} \leq \sqrt{\eta} \gamma\|\Delta(t)\|_{\infty} \approx 2.78$. A satisfactory state and unknown input estimation results are then obtained as shown in figures 2 and 3 which is also illustrated by the state estimation errors depicted in figure 4.

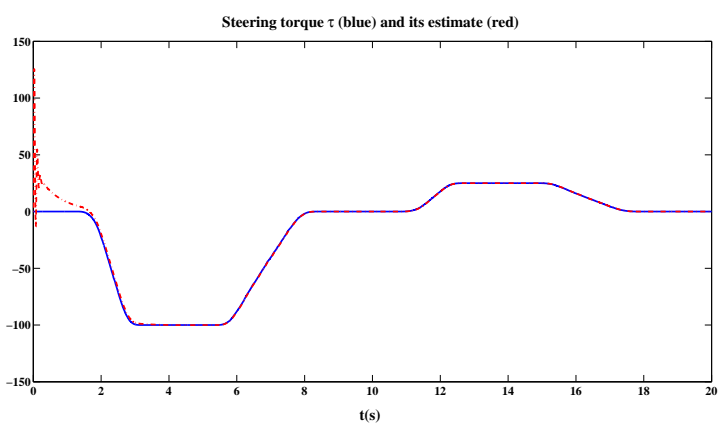

Fig. 2. Rider's torque (blue) and estimated torque (red)
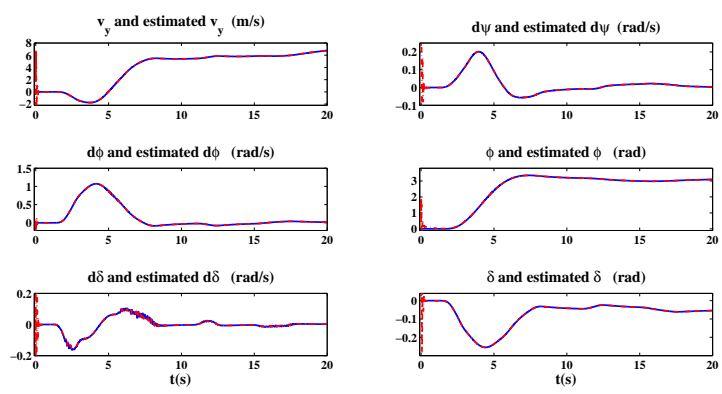

Fig. 3. Actual states (blue) and estimates states (red)

In order to test the observer in the presence of measurement noise, let us consider the same observer's parameters and assume that the measurement signals are affected by a centered and random noise with magnitude $5 \%$ of the maximal values of the measured variables. The obtained results are depicted in the figure 5, the states are then estimated accuratly. The estimation of the steering torque is also acceptable, but the effect of the measurement noise is visible. This is due to the presence of a derivation in the adaptation law leading to estimate the steering torque and also due to the large value of $\Gamma$. It is then possible to reduce the effect of the measurement noise by reducing the value of $\Gamma$. A compromize can then be obtaind by an adequate $\Gamma$ selected sufficiently large to ensure an accurate estimation of the unknown input and sufficiently less to reduce the effect of the measurement noise.

Of course the ISpS property is weaker than ISS or asymptotic stability, but the proposed observer with the estimation's

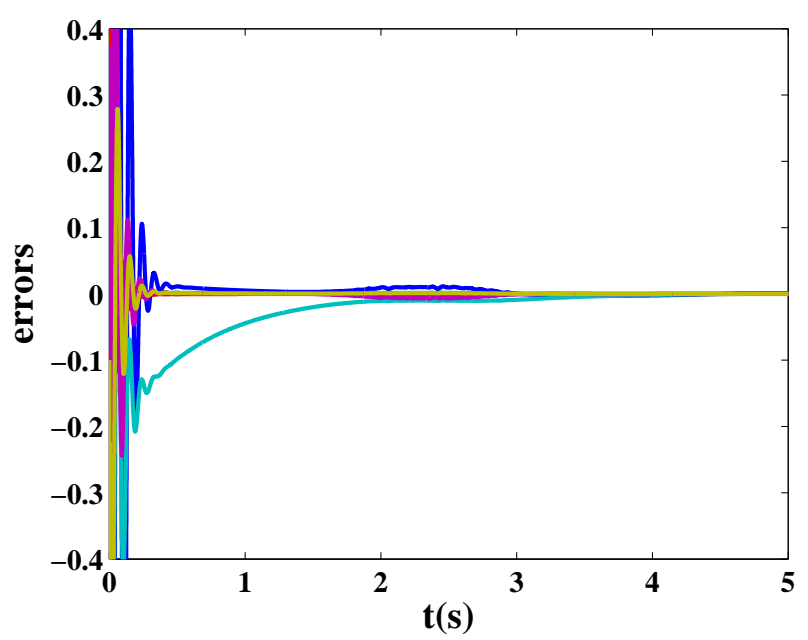

Fig. 4. State estimation errors on the time range $\left[\begin{array}{ll}0 & 5\end{array}\right](s)$
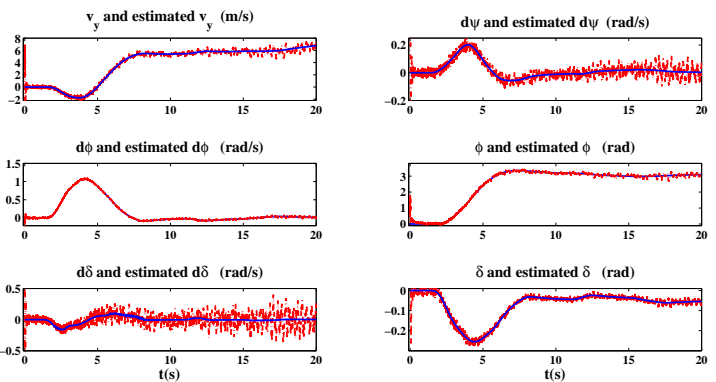

Fig. 5. Actual states (blue) and estimated states (red) in noised measurement situation

torque adaptation law can only guarantee the ISpS property. Nevertheless, the simulation results are satisfactory. Furthermore, some parameters in the proposed design approach are fixed $a$ priori, in future work, this will be treated in order to take them into account in the optimization problem leading to optimal values which give better results and less theoretic bounds values.

\section{CONCLUSION}

In this paper, an unknown input and state observer for estimating the lateral dynamics and the steering torque in motorcycles is proposed. A nonlinear model is then considered with some nonlinearities and time-varying longitudinal velocity. The proposed approach is based on the transformation of the nonlinear lateral dynamics model using sector nonlinearity transformation in Takagi-Sugeno structure, the specificity of this part is that the weighting functions of the T-S model depend on the state variables which are not totally measured. The study of this type of model is more difficult compared to T-S models with measured premise variables. Based on the obtained model, an adaptive observer is then proposed. The observer's convergence is studied using Lyapunov theory and LMI conditions are given to ease the design of the observer with dedicated softwares. It is pointed 


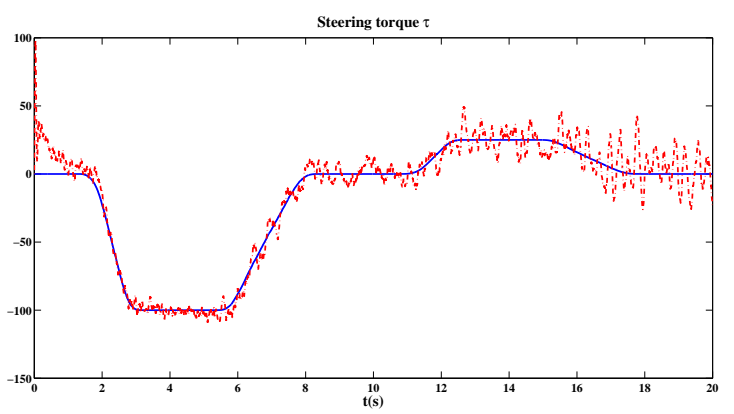

Fig. 6. Steering torque (blue) and estimated steering torque (red) in noised measurement situation

out that this observer guaranty the ISpS property. Simulation results are provided which illustrate the effectiveness of the proposed observer in estimating both the states and the steering torque. In future work, the T-S model will be refined by taking into account some other nonlinear behaviors, namely, the nonlinear form of the lateral forces (Pacejka's or Dugoff's model) and taking into account the longitudinal motion. The observer will be then redesigned for more accuracy. In addition, the lateral and longitudinal forces estimation will be considered in order to estimate the road adhesion. This is in the perspective to synthesis risk functions which inform the rider on dangerous situations. Some validation results in real situations will be published in future papers.

\section{ANNEX 1}

The components of the matrix $A(x(t))$ are defined as follows:

$$
\begin{aligned}
a_{11} & =-C_{f 1}-C_{r 1} \\
a_{12}\left(v_{x}\right) & =\frac{1}{v_{x}}\left(-C_{f 1} L_{f}+C_{r 1} L_{r}\right)-M v_{x} \\
a_{14} & =C_{f 2}+C_{r 2} \\
a_{15}\left(v_{x}\right) & =\frac{1}{v_{x}} \eta C_{f 1} \\
a_{16} & =C_{f 1} \cos (\varepsilon)+C_{f 2} \sin (\varepsilon) \\
a_{21} & =-C_{f 1} L_{f}+C_{r 1} L_{r} \\
a_{22}\left(v_{x}\right) & =\frac{1}{v_{x}}\left(-C_{f 1} L_{f}^{2}-C_{r 1} L_{r}^{2}\right)-M_{f} k v_{x} \\
a_{23}\left(v_{x}\right) & =\left(\left(\frac{i_{f y}}{R_{f}}\right)+\left(\frac{i_{r y}}{R_{r}}\right)\right) v_{x} \\
a_{24} & =C_{f 2} L_{f}-C_{r 2} L_{r} \\
a_{25}\left(v_{x}\right) & =\frac{1}{v_{x}} \eta L_{f} C_{f 1}+\left(\frac{i_{f y}}{R_{f}}\right) \sin (\varepsilon) v_{x} \\
a_{26} & =L_{f} C_{f 1} \cos (\varepsilon)+L_{f} C_{f 2} \sin (\varepsilon) \\
a_{32}\left(v_{x}, \dot{\phi}\right) & =-\frac{1}{v_{x}}\left(M_{f} j+M_{r} h+\left(\frac{i_{f y}}{R_{f}}\right)+\left(\frac{i_{r y}}{R_{r}}\right)\right) \\
& +\left(M_{f} j^{2}+M_{r} h^{2}+I_{f y}+I_{r y}-I_{r z}-I_{f z} \cos (\varepsilon)^{2}\right. \\
a_{34}(\phi) & =\left(M_{f y} \sin (\varepsilon)^{2}\right) \dot{\phi} \\
&
\end{aligned}
$$

$$
\begin{aligned}
a_{35}\left(v_{x}\right) & =\left(\frac{i_{f y}}{R_{f}}\right) \cos (\varepsilon) v_{x} \\
a_{36} & =M_{f} e g-\eta F_{z f} \\
a_{51}\left(v_{x}\right) & =\frac{1}{v_{x}} \eta C_{f 1} \\
a_{52}\left(v_{x}\right) & =\frac{1}{v_{x}} \eta L_{f} C_{f 1}-v_{x}\left(M_{f} e\right)\left(\frac{i_{f y}}{R_{f}}\right) \sin (\varepsilon) \\
a_{53}\left(v_{x}\right) & =\left(\frac{i_{f y}}{R_{f}}\right) \cos (\varepsilon) v_{x} \\
a_{53}(\phi) & =\left(M_{f} e g-\eta F_{z f}\right) \frac{\sin (\phi)}{\phi}-\eta C_{f 2} \\
a_{55}\left(v_{x}\right) & =\frac{\eta^{2} C_{f 1}}{v_{x}} \\
a_{56} & =-\eta C_{f 1} \cos (\varepsilon)-\eta C_{f 2} \sin (\varepsilon)+\left(M_{f} e g-\eta F_{z f}\right) \sin (\varepsilon) \\
e_{12} & =M_{f} k \\
e_{13} & =M_{f} j+M_{r} h \\
e_{15} & =M_{f} e \\
e_{22} & =M_{f} k^{2}+I_{r z}+I_{f x} \sin (\varepsilon)^{2}+I_{f z} \cos (\varepsilon)^{2} \\
e_{23} & =M_{f} j k+C_{r x z}+\left(I_{f z}+I_{f x}\right) \cos (\varepsilon) \sin (\varepsilon) \\
e_{25} & =M_{f} e k+I_{f z} \cos (\varepsilon) \\
e_{33} & =M_{f} j^{2}+M_{r} h^{2}+I_{r x}+I_{f x} \cos (\varepsilon)^{2}+I_{f z} \sin (\varepsilon)^{2} \\
e_{35} & =M_{f} e j+I_{f z} \sin (\varepsilon) \\
e_{55} & =M_{f} e^{2}+I_{f z}
\end{aligned}
$$

\begin{tabular}{|c|c|}
\hline$M_{f}$ & Mass of front frame \\
\hline$M_{r}$ & Mass of rear frame \\
\hline$j$ & $\begin{array}{l}\text { Distance between the center of gravity } \\
\text { of the front frame and ground }\end{array}$ \\
\hline$k$ & $\begin{array}{l}\text { Distance between the centers of gravity } \\
\text { of each frame }\end{array}$ \\
\hline$L_{f}$ & $\begin{array}{l}\text { Distance between the center of gravity } \\
\text { and the front wheel }\end{array}$ \\
\hline$L_{r}$ & $\begin{array}{l}\text { Distance between the center of gravity } \\
\text { and the rear wheel }\end{array}$ \\
\hline$e$ & $\begin{array}{l}\text { Distance between the fork and the center } \\
\text { of gravity }\end{array}$ \\
\hline$h$ & Height of the center of gravity \\
\hline$\varepsilon$ & Steering head angle \\
\hline$i_{f y}$ & Polar moment of inertia of front wheel \\
\hline$i_{r y}$ & Polar moment of inertia of rear wheel \\
\hline$I_{r z}$ & Camber inertia of rear wheel. \\
\hline$R_{f}$ & radius of the front wheel \\
\hline$R_{r}$ & radius of the rear wheel \\
\hline$g$ & Acceleration due to gravity. \\
\hline $\begin{array}{l}\sigma_{f} \\
M\end{array}$ & $\begin{array}{l}\text { Front and rear tire relaxation lengths respectively } \\
\text { total mass of the motorcycle } M=M_{f}+M_{r}\end{array}$ \\
\hline$C_{f 1} \quad C_{f 2}$ & $\begin{array}{l}\text { Front and rear tire cornering } \\
\text { stiffnesses respectively }\end{array}$ \\
\hline$C_{r 1} \quad C_{r 2}$ & Front and rear tire camber stiffnesses respectively \\
\hline & Pneumatic trail \\
\hline & Forward speed \\
\hline$\sigma_{r}, \quad \sigma_{f}$ & Front and rear tyre relaxation lengths respectively \\
\hline & road's curvature \\
\hline
\end{tabular}

\section{ANNEX 2}

\section{REFERENCES}

[1] A. Akhenak, M. Chadli, J. Ragot, and D. Maquin. Design of sliding mode unknown input observer for uncertain Takagi-Sugeno model. In 15th Mediterranean Conference on Control and Automation, MED'07, Athens, Greece, 2007. 
[2] B. Amans and M. Moutreuil. Rider project . research on accidents involving powered two-wheelers final report $\mathrm{n}$ rider200503-10. Technical report, French National Agency of Reaserch, 2005.

[3] P. Bergsten, R. Palm, and D. Driankov. Observers for Takagi-Sugeno fuzzy systems. IEEE Transactions on Systems, Man, and Cybernetics - Part B: Cybernetics, 32(1):114-121, 2002.

[4] I. Boniolo and SM Savaresi. Estimate of the lean angle of motorcycles. Technical report, ISBN 978-3-639-26328-2, VDM Verlag, Germany, 2010.

[5] V. Cossalter and R. Lot. A motorcycle multibody model for real time simulation based on the natural coordinates approach. Vehicle System Dynamics, 37(6):423-447, 2002

[6] P. De Filippi, M. Corno, M. Tanelli, and S. Savaresi. Singlesensor control strategies for semi-active steering damper control in two-wheeled vehicles. Vehicular Technology, IEEE Transactions on, 61(2):813-820, February 2011.

[7] B. Evangelos. Saferider project. final report $\mathrm{n}$ rider200503-10, Technical report, French National Agency of Reaserch, 2010.

[8] S. Hima, L. Nehaoua, N. Seguy, and H. Arioui. Suitable two wheeled vehicle dynamics synthesis for interactive motorcycle simulator. In In Proc. of the IFAC World Congress, pages 96-101, Seoul, Korea,, July 6-11 2008.

[9] D. Ichalal, B. Marx, J. Ragot, and D. Maquin. Fault tolerant control for Takagi-Sugeno systems with unmeasurable premise variables by trajectory tracking. In IEEE International workshop on Industrial Electronics, ISIE'10, Bari, Italy, July 4-7 2010.

[10] D. Ichalal, B. Marx, J. Ragot, and D. Maquin. State estimation of Takagi-Sugeno systems with unmeasurable premise variables. IET Control Theory \& Applications, 4(5):897-908, 2010.

[11] Z.P. Jiang, A.R. Teel, and L. Praly. Small gain theorem for ISS systems and applications. Journal of Math. Control Signals Systems, 7, 1994.

[12] M. Lazar, D. Munoz de la Pena, W.P.M.H Heemels, and T. Alamo. On input-to-state stability of minmax nonlinear model predictive control. Systems \& Control Letters, 57, 2008.

[13] French Interministerial National Observatory of Road Safety. characteristics and causes of road accidents. Technical report, 2010.

[14] R.S. Sharp. The stability and control of motorcycle. Journal of Mechanic Engeeniering and Science, 13(5):316-329, 1971.

[15] H. Slimi, H. Arioui, L. Nouveliere, and S. Mammar. Advanced motorcycle-infrastructure-driver roll angle profile for loss control prevention. In Intelligent Transportation Systems, 2009. ITSC '09. 12th International IEEE Conference on, pages 1 -6, oct. 2009.

[16] T. Takagi and M. Sugeno. Fuzzy identification of systems and its applications to modeling and control. IEEE Transactions on Systems, Man, and Cybernetics, 15:116-132, 1985.

[17] K. Tanaka and H.O. Wang. Fuzzy Control Systems Design and Analysis: A Linear Matrix Inequality Approach. John Wiley and Sons, 2001.

[18] AP Teerhuis and STH Jansen. Motorcycle state estimation for lateral dynamics. Bicycle and Motorcycle Dynamics, 2010.

[19] H. Wang and S. Daley. Actuator fault diagnosis: an adaptive observerbased technique. IEEE Transactions on Automatic Control, 41:10731078, 1996.

[20] J. Yoneyama. $H_{\infty}$ filtering for fuzzy systems with immeasurable premise variables: an uncertain system approach. Fuzzy Sets and Systems, 160(12):1738-1748, 2009. 